\title{
A game prototype for understanding the safety issues of a lifeboat launch
}

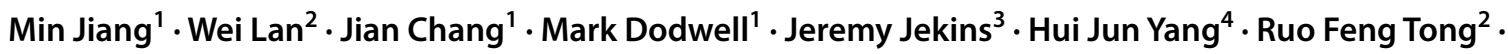 \\ Jian Jun Zhang ${ }^{1}$
}

Received: 9 December 2016 / Accepted: 14 January 2018 / Published online: 23 January 2018

(c) The Author(s) 2018. This article is an open access publication

\begin{abstract}
Novel, advanced game techniques provide us with new possibilities to mimic a complicated training process, with the added benefit of enhanced safety. In this paper, we design and implement a 3D game with the support of virtual reality equipment which imitates the process of a lifeboat launch, involving both tractor manoeuvres and boat operations. It is a complex but vital process which can save lives at sea but also has many potential hazards. The primary objective of the game is to allow novices to better understand the sequence of the operations and manage the potential risks which may occur during the launch process. Additionally, the game has been promoted to the general public for educational purposes and to raise awareness of the safety issues involved. The key modules of the game are designed based on physical simulations to give the players enhanced plausible cognition and enjoyable interaction. We conducted two case studies for the two purposes of the games: one for training with volunteers without launching experience and the other for public awareness of the potential hazards with young children. The game is proven to be very promising for future professional training, and it serves the educational purpose of awareness of the safety issues for general public while being entertaining.
\end{abstract}

Keywords Serious games $\cdot$ Virtual reality $\cdot$ Lifeboat launch $\cdot$ Game-based training $\cdot$ General public

\section{Introduction}

Two out of three people in the UK will have seaside activities at least once a year. Every year around 7000 of them will face serious difficulties. Lifeboat volunteers are highly trained for lifesaving, and they are ready to respond to any emergency at sea $24 \mathrm{~h}$ a day (RNLI 2017a). These rescues normally involve lifeboat launching which becomes such a vital problem. However, to the best of our knowledge, this is the best paper of a lifeboat launch simulator for training purposes.

A lifeboat launch is a complicated process which involves the collaboration and synchronisation of several

Jian Chang

jchang@bournemouth.ac.uk

1 National Centre for Computer Animation, Bournemouth University, Poole, UK

2 Zhejiang University, Hangzhou, China

3 Royal National Lifeboat Institution, Poole, UK

4 Northwest A\&F University, Yangling, China roles, including that of the tractor driver, the boat crew and the inspector. Their cooperation has a direct impact on the results of the rescues. Familiarity with the operations greatly affects the speed and effectiveness of the rescue. The rescuers themselves may also face severe conditions, e.g. darkness, tides, wind, and rain or snow. Fully understanding the launch process and adequate practice become especially important before facing the real-life danger. Therefore, an advanced training process is vital (Fig. 1).

Traditionally, the training procedure for novices is to have them read the instructional manuals and then practice in a real launch scenario. However, there are almost always risks for inexperienced operators in those situations. Studying a manual of a complicated operation process can be hard for beginners to understand, especially when the process involves multiple operators with different roles. The emergence of game and virtual reality (VR) techniques (Brooks 1999; Burdea and Coiffet 2003; Shermann and Craig 2003) provides us with a new and effective platform to simulate the launch process which can then be used for training, while avoiding the associated risks. In this paper, we develop 


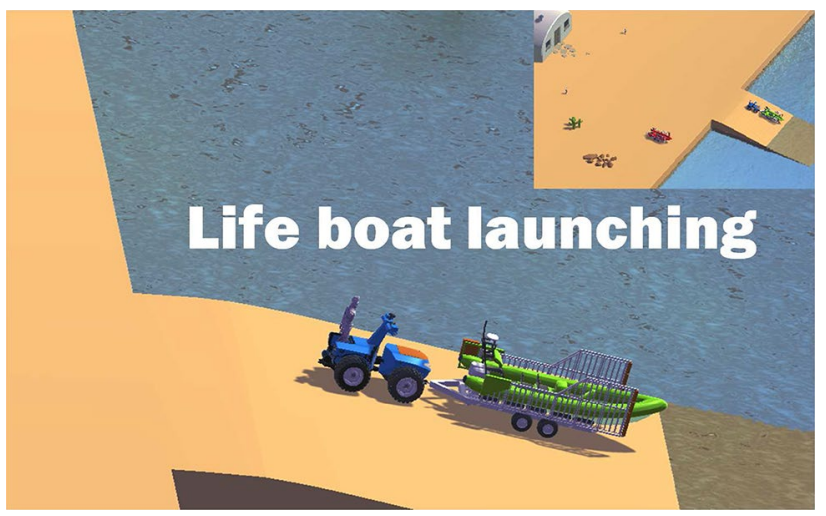

Fig. 1 Game overview

conceptual and technical approaches to build a 3D game prototype for a lifeboat launch simulator.

Game-based training for a lifeboat launch confers a wide range of benefits for both academia and industry (Yann 2016), including:

1. Providing an interactive virtual environment for the trainees, where they can play different roles and get a complete picture of the launch process.

2. The ability to simulate dangerous or risky situations, some of which may occur rarely in the real world. Most importantly, it also allows the trainees to choose the level of training based on their confidence and without risks to their lives.

3. It provides remarkable time and costs savings for training and caters to remote training where trainees are not necessarily on site. It also supports peer review, feedback and training assessment.

4. It makes learning enjoyable. Since the lifeboat launch process is not widely known to the public, it will also encourage public engagement for educational purposes.

Our game is based on the combination of simple functional modules, and each of which is responsible for a basic task. The key modules are implemented based on physical laws, which enhances the user experience during the game playing by giving lifelike feedbacks of the manoeuvering. The realistic physics gives players cognitive correctness and allows them to understand and operate the lifeboat launching more easily. Some of the other modules are heuristically designed. Since the launch process involves moving both on land and over water, we will discuss how the different frictional forces (due to different road surface conditions) impact the manoeuvres of the tractor. The tractor-trailer connection also affects the behaviour of the tractor. Finally, we demonstrate how to find the proper water level for the boat launch. Distractions are purposely introduced to challenge and train the players' recognition of the potential hazards. The prototype provides the possibility to customise the function of each module as required.

We use the Unity Game Engine (Unity 2017) to build the platform and a HTC Vive virtual reality system to enhance the immersion and interaction of the game. The game evaluates the performance of the players based on a score. Finishing tasks over the prescribed target time will lose the game, and the algorithm penalises players when they mistakenly operate the sequence of the training process or make any unsafe operations, e.g. causing a collision or turning over. A good winning strategy for the game is to simply finish the tasks as fast as possible while avoiding unsafe operations.

The primary objective of this work is to design, implement and test the architecture enabling a complete lifeboat launch process. This work has two main contributions:

- A novel game-based training system to familiarise beginners with the lifeboat launch process, without the risks of the real world.

- A game platform to encourage public engagement for the purpose of safety education.

\section{Related work}

There is little doubt that computer games for entertainment are influencing our lives. However, it was not until 2003 that Gee highlighted the benefits of video games for learning (Gee 2003).

Knowledge cannot be transmitted but is actively built. It is normally individually constructed and socially coconstructed by learners based on their interpretations of experiences in the real world. Therefore, instruction learning should consist of creating situations that provide interpretable experiences (Jonassen 1999). However, sometimes these situations are impossible to be recreated in the real world due to costs, safety, time or resource availability.

The emergence of virtual reality technology enables digital simulations of extreme situations in which learners can practice difficult, exacting, life threatening or mission-critical skills (Epper et al. 2012). It engages through rich visuals that entice learners into fantasy worlds and at the same time permeate designed learning knowledge. However, simulations by themselves lack an intrinsic competitive element which is the hallmark of a game. Therefore, game-based learning integrated with simulation has attracted the attention of researchers.

Games for educating, training and information are defined as Serious Games (Abt 1987). Serious games allow players to experience a "real-life" situation in an artificial environment. Many researchers and educational practitioners believe that serious games offer strong benefits in education 
and training through their experiential interactions (Gredler 2004; Backlund and Hendrix 2013; Youngblut 1998).

Playing can be a good motivator for those who have to learn skills. The entertainment dimension highlights the emotional experience, which increases the attractiveness for all ages, of the game beyond its educational aim. For these reasons, games can be considered powerful tools for training.

Game-based training alleviates the burden of instructional material and reduces the workload of the instructors. Due to high interactivity, these computer-based training methods make the learning process more fun and enjoyable (Greitzer et al. 2007). The use of simulation systems demonstrates the possibilities for the development of different skills (CGM 1994; Schneider 1985; Michael and Chen 2005; Susi et al. 2007). In the view of Gredler (2004), game-based training bridges the gap between the classroom and the real world by providing experience with complex, evolving problems, which is the case in a lifeboat launch training. Gamberini et al. (2008) proved the relations between playing games and training cognitive abilities.

Fery and Ponserre (2001) analysed a golf game used to learn the real game of golf. The golf game showed a positive transfer of skills from the virtual to real settings. Rosser et al. (2004) emphasised how video games could be successfully used in the training of laparoscopic surgeons which requires high levels of visual attention, manual dexterity and hand-eye coordination. Some typical training game applications are those targeting specific professional skills for military personnel, such as aircraft pilots. Gopher et al. (1994) observed that cadets trained with a computer game performed significantly better in flight sessions than those trained with traditional methods. As a consequence, the Israeli Air Force incorporated the game into the regular training programme of its pilots. In this case, the enhancement in perceptual and cognitive processing could induce significant differences in job performance. Therefore, we have designed the lifeboat launch game platform for the purpose of professional training.

Many educational simulation systems emphasise the importance of physical factors to facilitate the training process. For example, car racing games achieve extremely immersive user experiences by means of game controllers which consist of realistic real-time force feedback steering wheels and gear shifts. Oztel and Oz (2014) simulated a virtual driving platform for educational purposes which contained all the necessary hardware and software modules. Such "vehicle-centred" simulators emphasise the simulation of the physical motion and sense. These simulators were designed to train learners to control the vehicle. Different from "vehicle-centred" approaches, our game is a kind of "traffic-centred" simulator, which includes but is not limited to vehicle manoeuvres. Our design focuses on high-level skills such as recognising situations and developing strategies to avoid potential hazards. At the same time, understanding the working condition of a tractor and a trailer in a lifeboat launch is also important.

Virtual environments can be also exploited for safety training in emergency situations. There exists advanced simulators for traffic safety research and driving education (Lebram 2006; Östlund et al. 2006). Gamberini et al. (2010) showed that in these situations users could show changes to their behaviour, in the direction of an increase in the speed of their escape at the detriment of the movements' precision. In this paper, we have also designed the game for the purpose of general public engagement. We elaborately design a series of scenarios with potential hazards during the launch process, which can subconsciously encourage the public to understand and avoid these hazards, e.g. running across a field near the launch site.

Driving simulators may consist of various different pieces of equipment. For example, some simulators are controlled with a keyboard, while others use a steering wheel. Furthermore, real cars can be used as simulators to increase the realism (Lebram 2006). However, these kinds of simulation systems are very expensive, and the training programmes are typically formal, structured and intensive (Wolffelaar et al. 1999). In this paper, we focus on presenting a 3D virtual simulation environment of a lifeboat launch game using either keyboard or VR controller input. The results of some initial evaluation are also presented.

\section{Game design}

In this section, we describe the game design based on Salen and Zimmerman (2004) which involves the pedagogical considerations of meaningful play and interactivity. The game pipeline specifies the key operations during the lifeboat launch process. The virtual environment settings allow the players to choose the different levels according to their own confidence.

\subsection{Game objectives and target users}

This novel design of the game allows two levels of engagement: one is for the professional users in lifeboat launch training, and the other is for safety awareness education of the general public. The storyline is the same for both, but the implementations are different for these two targets. Our game consists of multiple functional modules each responsible for a simple task. Thus, the game is customisable for approaching both targets. For training purposes, we focus on the correct sequence of the lifeboat launch process, while for general public engagement we emphasise the immersion and fun of the game. 
Based on the above considerations, there are several aspects we have considered in our game design, and these are summarised below:

\section{For Training Purposes:}

- The sequence of operations is very important. For a fuller understanding, each step of the operation maps directly to the instruction manual.

- The training system aims to enhance the users' familiarity of the operations and improve the efficiency under the premise of safety.

- The game inspects the users' abilities to handle hazards and their responses to emergencies;

- The game requires the understanding of the difference of road surface conditions in order to better manipulate the vehicle.

\section{For the General Public:}

- The process transits into a game that simply asks players to complete the task without the need of a deeper understanding of the process.

- The cognition of the potential hazards is what matters the most.

- The players do not necessarily understand the exact sequence of the launch operations.

- The game promotes enjoyability where appropriate.

\subsection{Game pipeline and system architecture}

To serve both purposes as discussed above, we design the game pipeline according to the actual launching manual, as shown below:

1. * The tractor connects with the lifeboat: make sure it is connected properly.

2. The inspector checks the safety of the environment and that the tractor working correctly.

3. * The tractor driver and boat crew embark.

4. The tractor driver, boat crew and inspector establish radio communications.

5. The inspector gives the launch signal: make sure the signal is received before launching.

6. * The tractor moves out of the boathouse: make sure not to hit or collide with any of the environmental assets.

7. * The tractor drives towards the seaside: choose the correct driving mode according to the different road conditions.

8. * The tractor drives into the water: adjust the sensors, cameras and lights to match the different environmental conditions.
9. * The tractor drives to the launch area: reach the launch point and avoid the surrounding under water hazards.

10. * The tractor disconnects with the lifeboat when the water depth is adequate for launching: detect the right water level.

11. * The tractor drives back to the boathouse: adjust the driving mode and make sure to avoid any hazards.

12. * The tractor driver disembarks.

For training purposes, all of the operations above must be completed in the right order. Any mistake in the sequence will incur penalty points, details of which can be found in Sec. 3.3. The training instructor can set the target completion time for trainees to practice and improve their launch efficiency. Pull-down menus are designed for each step of the operations to emphasise the orders of operations, as shown in Fig. 2.

For the public engagement, we only expect the players to fulfil the steps marked with *. In order to guarantee the user experience, the operations are designed purposely for public engagement, e.g. steps $2,4,5$, which are not associated with particular visual feedback in the game, are opted out. However, they are still very important for training purposes. There are designed hazards during the launch process to make the game entertaining, e.g. a running dog, rolling balls and pedestrians. Players should always be cautious about these hazards and move safely without incurring any danger. This is a good way to effectively train the players' response to and awareness of hazards. The operation sequence checks are disabled here to reduce the game difficulty. The players will only rely on the keyboard or VR controller as the input without the use of pull-down menus.

There are three main assets in this game: characters (tractor driver, boat crew and inspector), tractors and boats. We control the motion of the character, using a pre-recorded animation, e.g. idle, walk and embark/disembark. Once the

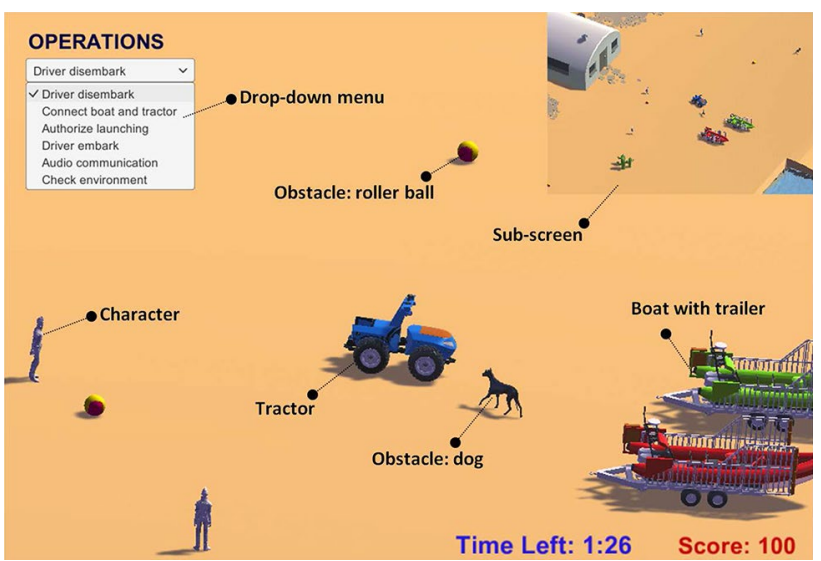

Fig. 2 Main interface and menu of selecting operations 
driver embarks the tractor, the driver is parented with the tractor which means we only need to manoeuvre the tractor. The manoeuvres of the tractor will be directly controlled using either the keyboard or VR controller, and the same principle applies to the boat crew and the boat. The boat is pulled by the tractor and affected by the water. The relationship between them is shown in Fig. 3.

The player manoeuvres the tractor, controls the characters and fulfils the launch process without incurring any danger within a given time. To make the game more immersive and enjoyable, both background and interactive music is added, such as tractor engine sound, collision sound and sea wave sound. The score system gives feedback at the end of each game.

\subsection{Score system}

The maximum score in the game is 100 . If the player runs out of time, he or she loses. Each incorrect operation, violation or any unsafe behaviours incurred, triggers certain penalty points. The details of the penalty points for the different violation scenarios are listed in Table 1 . Penalty points for each item can be superimposed. For example, two collisions with environmental assets will incur 60 penalty points. If the score is reduced to 0 or less, the player loses the round and

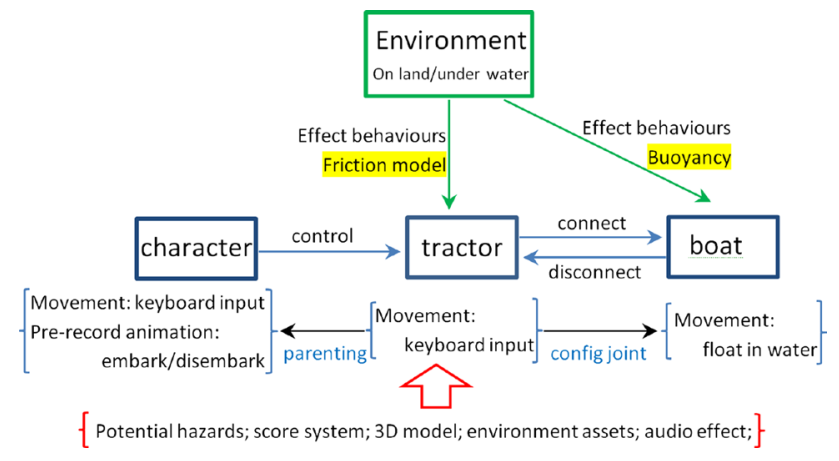

Fig. 3 System architecture can choose to replay it. The possible outcomes of the game are shown in Fig. 4.

\subsection{Level setting}

An individual trainee starts with a standard setting and a sufficient target time. The instructor monitors the performance of the trainee. If the trainee can complete the standard scenarios without mistakes and within a decent time, the system brings him to new scenarios with increased difficulty. If an error is committed, feedback is presented on the screen to the trainee, and the trainee may need to go through the same scenario repeatedly until he or she succeeds.

We design three levels for the game: a higher level indicates more hazards and shorter time. The level setting allows players to familiarise themselves with the process of dealing with hazards and to finally reach the point of proficiency. This level feature of the game allows the instructors and trainees to choose the proper level according to their

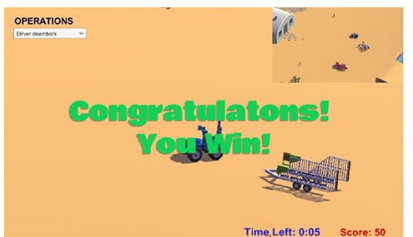

(a)

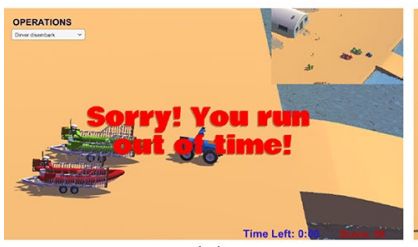

(c)

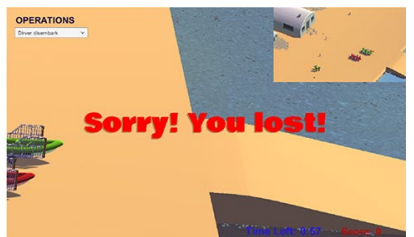

(b)

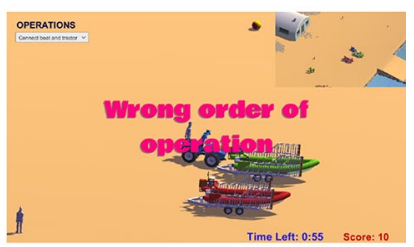

(d)
Fig. 4 Various outcomes. a Win by fulfilling the tasks within the limited time and while maintaining a positive score. $\mathbf{b}$ Lose with a score of 0 . c Lose due to running out of time. d Failure to comply with the correct sequence of operations, play can continue as the score remains positive and there is time left
Table 1 The guide of violations and penalty points in our game

\begin{tabular}{lc}
\hline Violations & Penalty points \\
\hline Failure to comply with the sequence of operations & 40 \\
Boat does not properly connect with the tractor & 40 \\
Tractor collides with an environmental asset & 30 \\
Driver or crew do not safely embark/disembark & 30 \\
Wrong driving model is used for the different road conditions & 30 \\
Collision with a human or a hazard (moving vehicle, animal) & 50 \\
Tractor loses control and falls into the water & 100 \\
Boat loses control or is not properly released into the water & 100 \\
Boat collides with an environmental asset in the water & 30
\end{tabular}


experience which avoids the situation where it is too difficult for a novice or a waste of time for experienced users.

\section{Virtual reality development}

To enhance the immersion of this game, we alternatively combine the game with a VR headset. This VR technology allows users to physically manipulate the virtual space, and control the crew walking, embarking, driving and the boat launch. Simultaneously, the instructor can use the computer display to monitor the progress.

Compared to simulators with wheels or something closer to a real tractor, VR headset and controls have several advantages:

1. VR headset can provide a virtual environment for the players, who can experience different beach conditions, e.g. obstacles and water levels.

2. VR controller allows the players different roles during the game, not only tractor driver, but also boat crew and indicator, which provide players a complete picture of the whole launch process.

3. VR game is easier to set up than to have a real tractor on site which might occupy a lot of space.

4. the functional operation of VR controller can be changed easily if new modules are added into the game, while the actual tractor/wheel simulators are not as flexible.

Since this is the first approximation, we choose a VR headset and controls for the game simulator, and other controls are part of future work. The HTC Vive, a VR head-mounted display with motion sensors which makes the experience more real-like, is used in this work for the VR development. It is well supported by Unity (5th Edition) plug-ins. HTC Vive consists of three main components: an adjustable headset, two base stations and two wireless controllers. The exact location and orientation of the headset and controllers can be tracked in space. These two controllers enable the users to interact with the 3D assets (characters, tractors and lifeboats) naturally and intuitively in the virtual environment. The headset is used to control the camera view in the VR game. We use the first person view in the VR game since the first person view is immersive and the third person view can cause dizziness since the motion is seen but not felt.

Once the game starts, the viewpoint follows the movement of headset in the real world. The user plays the role of the tractor driver and launches the lifeboat. In order to improve the user experience, UI information such as time, score and text messages are added to the headset display without affecting the game play. The manoeuvre of the tractor is directly guided using the Vive controllers as input. We use the touchpad of the Vives controllers as a joystick to control the manoeuvre when the character is walking and driving. Trigger, grip and menu buttons are mapped to the processes of the lifeboat launch, character embarking and lifeboat connecting. To conform with the non-VR game, once the driver embarks on the tractor, the driver is attached to the tractor which means the relative movement between the driver and the tractor is ignored. The structure of this VR game with the Vive is shown in Fig. 5.

Figure 6 shows the experimental examples with the game running, while the player is controlling the character or tractor.

During the game play, the users are immersed in an emergency scenario which requires a lifeboat launch. The use of VR equipment gives the users real-time feedback and real-like experiences that can be advantageous for their training. At the same time, the supervisor can monitor their training process to improve the training efficiency. Although we use the first person view in VR, the prolonged use of a VR device is not recommended due to dizziness. So far, the environment and assets are graphically primitive, but no complaints about this were received from the test users.

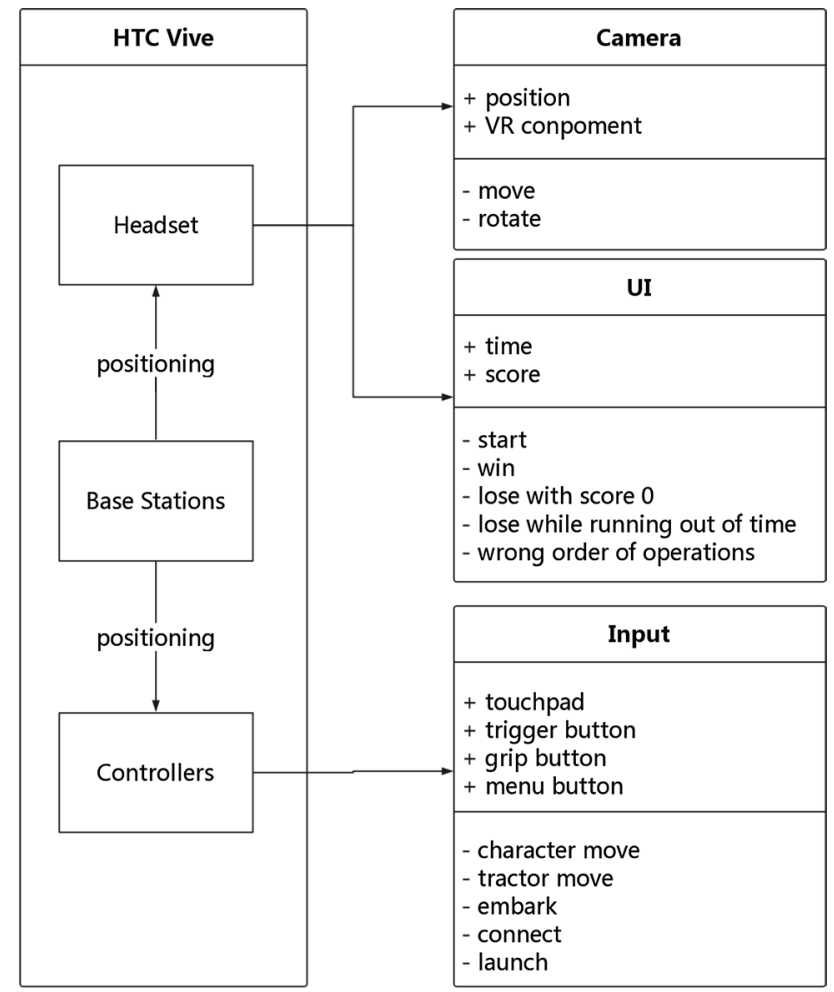

Fig. 5 The architecture of the VR game 


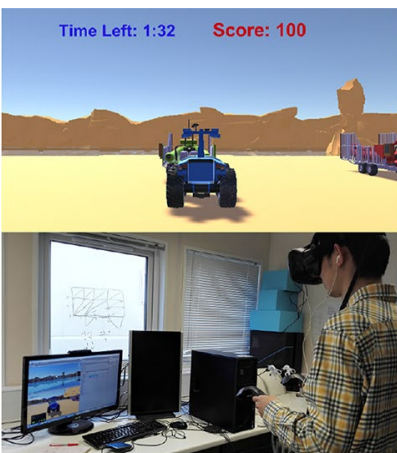

(a)

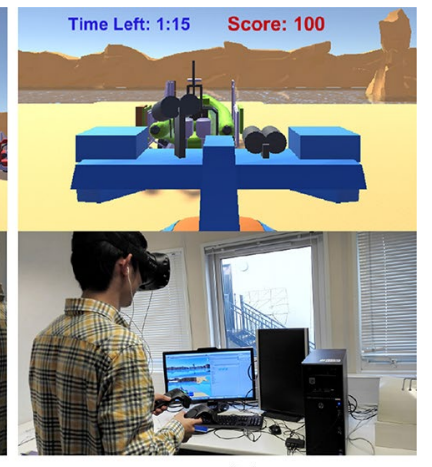

(b)
Fig. 6 Experimental examples. a A crew walking towards the tractor. b A crew driving the tractor

\section{Game physics}

In order to demonstrate the feasibility of our design, we implement a prototype system using Unity with C\#. The implementation of the game follows on physical laws, which enhances the user experience during the game playing by giving lifelike feedbacks of the manoeuvering. The players stated that the realistic physics allows them to understand and operate the lifeboat launching more easily.

\subsection{Motion control with rolling resistance}

In this game, the key motion control is the tractor manoeuvre on different types of terrains. To allow the players to have lifelike feedback, our game needs to simulate the different driving experiences.

Considering a constant pulling force, the speed of the tractor is mainly affected by the friction force of a vehicle tyre rolling on a road, often called rolling resistance. It can be calculated by a simplified model (Hibbeler 2007): $\mathbf{F}_{f}=C_{r r} \mathbf{W}$, where $\mathbf{W}$ is the load per wheel of the tractor. $C_{r r}$ is the rolling resistance coefficient. The direction of the rolling resistance force is opposing to the motion of the wheel.

In our game, the tractor will encounter various road conditions, involving a concrete road, sand, pebbles and seawater, as shown in Fig. 7. With different $C_{r r}$, the players can sense the various grounds changing when manoeuvring the tractor, giving a more immersive environment. The manoeuvre of the tractor is scripted in $\mathrm{C \#}$.

In Fig. 7(d), where the tractor is submerged in water, we need to consider the buoyancy force $\mathbf{F}_{b}$. The resistance from the water is related to the submerged height of the tractor $h$ and the relative velocity between the water $\mathbf{v}_{w}$ and the tractor $\mathbf{v}_{t}$. The overall rolling resistance can then be written as:

$\mathbf{F}_{f}=C_{r r}\left(\mathbf{W}-\mathbf{F}_{b}\right)+C_{w}|| \mathbf{v}_{t}-\mathbf{v}_{w} \| h$

where $C_{w}=0.002$ is the water resistance coefficient.

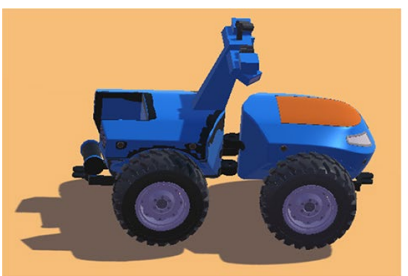

(a)

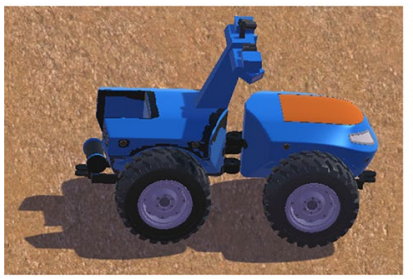

(c)

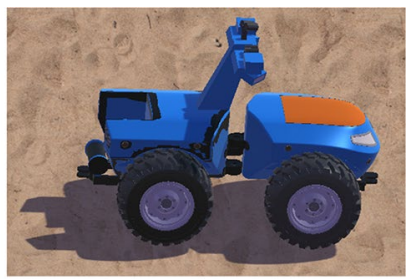

(b)

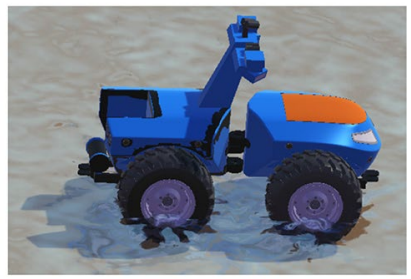

(d)
Fig. 7 Various road surface conditions. $C_{r r}$ is set according to references and common sense, e.g. seawater as a lubricated effect on the rolling resistance force. a A perfect concrete road $C_{r r}=0.01$ (Gillespie 1992). b A sand beach $C_{r r}=0.1$ (Gillespie 1992). c A pebble surface $C_{r r}=0.0385$ (Baker 1919). d A road surface submerged in seawater. $C_{r r}=0.005$

The basic manoeuvres of the vehicle are forward, backward and turning. When the tractor is turning an angle of $\theta$ shown in Fig. 8, there will be additional frictional force in the horizontal direction and the resultant friction force becomes $\mathbf{F}_{f} / \cos \theta$. Thus, the actual turning velocity of the tractor will become $\mathbf{v}_{0} \cos \theta$, where $\mathbf{v}_{0}$ is the normal forward velocity.

\subsection{Tractor-trailer hitching}

The load of tractor determines its rolling resistance. The load depends on the weight of the tractor itself as well as the implement weight (if any) that is carried by the tractor (Macmillan 2002). Therefore, when the boat is connected,

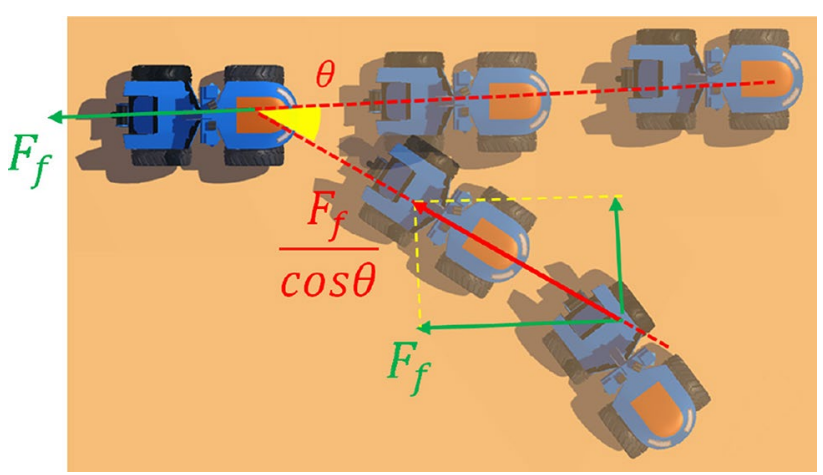

Fig. 8 Tractor turning conditions 
the tractor is performing slower since there is more weight on the wheels.

The hitching configurations between the tractor and the trailer not only limit the movement of the trailer, but also affect the behaviour of tractor (Aloni and Khedkar 2012).

In real life, the trailer is free to move in both the horizontal and vertical planes. We use a configuration joint in Unity for the hitching the tractor and trailer. The configuration joint is scripted to connect and disconnect the tractor and the boat to meet the mechanical requirements in both planes:

(i) The horizontal plane: the steering of the tractor has a joint effect on the turning of the trailer, as shown in Fig. 9. With the power force of the tractor $\mathbf{F}_{p}$ and the angular difference $\alpha$, the applied forward force exerted on the boat will be $\mathbf{F}_{p} \cos \alpha$. If $\alpha$ is too large ( $90^{\circ}$ in an extreme case), $\cos \alpha$ tends towards 0 , and the boat will experience little forward force, leading to difficulty with movement. Therefore, we limit $\alpha$ to be within $30^{\circ}$ to avoid unnecessary difficulties of the tractor movement.

The resulting rolling resistance force $\mathbf{F}_{f_{t}}$ can be calculated from the resistance force of the tractor $\mathbf{F}_{f_{1}}$ and the boat $\mathbf{F}_{f_{2}}$ as Eq. 2 (Fig. 10).

$\mathbf{F}_{f_{t}}=\sqrt{\mathbf{F}_{f_{1}}^{2}+\mathbf{F}_{f_{2}}^{2}+2 \mathbf{F}_{f_{1}} \mathbf{F}_{f_{2}} \cos \alpha}$

(ii) The vertical longitudinal plane: the tractor and trailer can have different directions on the vertical plane, e.g. on a slope in Fig. 11. Due to the gravity, there is an angle between the direction of the tractor and trailer $\beta$. Similarly, the drag force of the boat will be $\mathbf{F}_{p} \cos \beta$. We limit $\beta$ within $30^{\circ}$ as well to avoid no drag force on the boat. The total resistance force can be similarly calculated using Eq. 2 .

\subsection{Water level and buoyancy}

We calculate the amount of buoyancy to determine the water level for the boat release criterion. The boat will float when the buoyancy $\mathbf{F}_{b}$ force is equal to the force of gravity on the boat $G$. As long as the boat model is given, we can calculate

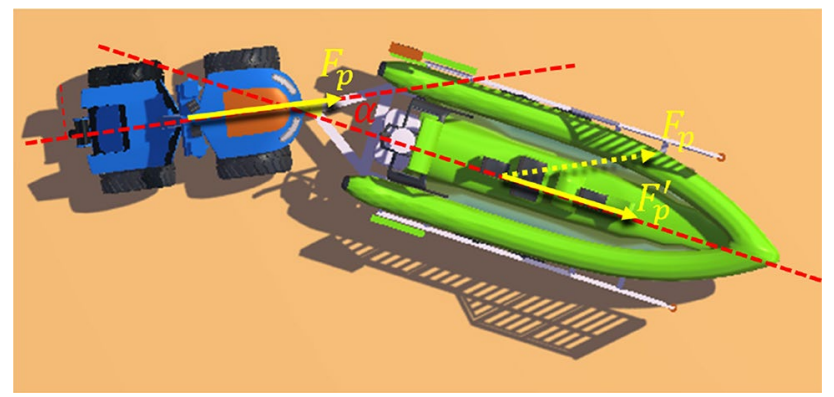

Fig. 9 Kinematic scheme of applied force, horizontal plane

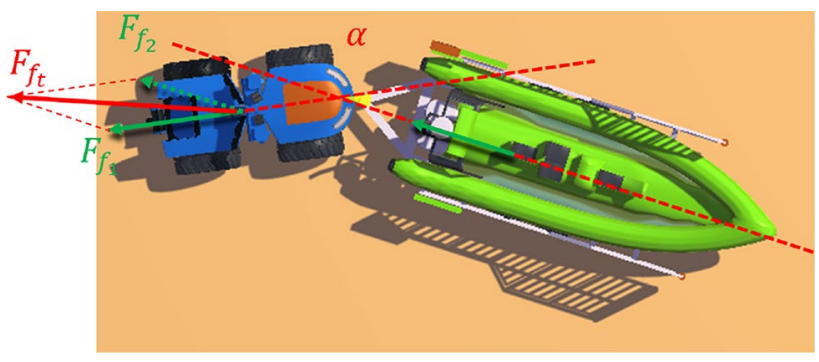

Fig. 10 Resulting rolling resistance forces

the height of the submerged volume $h$. Once the water level is larger than $h$, the boat is scripted to release.

It is noted that all the formulae of this model is a rough approximation to the real physics. We have to sacrifice the accuracy to accommodate the limitation of computing resources and request for real-time response. There is far more accurate simulation in engineering mechanics (Hibbeler 2007).

\section{User evaluation}

Our aim is to collect evidence on whether playing the game helps the players familiarise themselves with the lifeboat launch process and improve the safety awareness for the general public. We conducted two case studies for the two purposes of the games: one for training and the other for public awareness of the potential hazards. Both of the experiments followed the principles of the Bournemouth University's research ethics policy, and a written consent was obtained from each participant. We conducted mixed methods for the studies. On one side, we analyse the results of the experimental games based on marks and correctness; on the other side, we interview players after their case studies to encourage them to give us more information about the user experiences. These mixed methods allow us to receive more perspective from the game and simulator design.

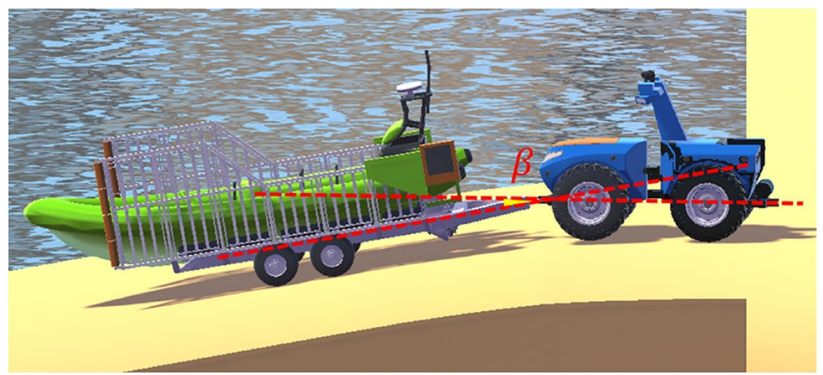

Fig. 11 Kinematic scheme, yaw plane 


\section{Study 1: For Training}

We recruited 20 volunteers (10 males and 10 females) for this case study, aged 23 to 41 . None of the participants had any prior lifeboat launch experience. For the purpose of comparative case studies, we separated the participants into two groups. Each group had 10 participants (5 males and 5 females).

Participants in Group One were instructed to play our game with a textual instructional manual for lifeboat launching, while participants in Group Two only studied the same textual manual without playing the game. The content of the manual is similar to the process described in Sec. 3.2 (12-step process). The game is closely designed based on the manual. Therefore, the game and the manual are actually conveying the same information, but in different formats. This maintains the fairness of the comparison since Group One is not getting extra more information than Group Two. Both groups were given the same time to review the manual or play the game. Since none of the volunteers has the experience of lifeboat launching, we found $30 \mathrm{~min}$ to be a fair amount time for them to go through the whole process. An interesting discovery was found that, for Group One, volunteers tend to spend most of the time playing the game and only take the manual as a reference when they are not clear about the next step, while for Group Two, volunteers have to hold the manual to try to understand the launching process. Afterwards, we tested their familiarity with the lifeboat launch process by giving the participants an unordered list of the launch operations. The participants were asked to reorder them in a limited time. The participants were tested with 5 trials, each with the same time limit. The average accuracy of each group for each time limit is shown in Fig. 13.

The results show that the mean value of the correctness rate of Group One is $91 \%$, while the Group two is $71 \%$ over 5 trials, and the standard deviation value of the correctness rate of Group is 10.84, while the Group two is 22.75. There is no significant difference between the performance of the male and female participants. Male participants tend to have a better start of 5.6\% superiority. However, it is quickly taken over by trials and female participants finish better than male

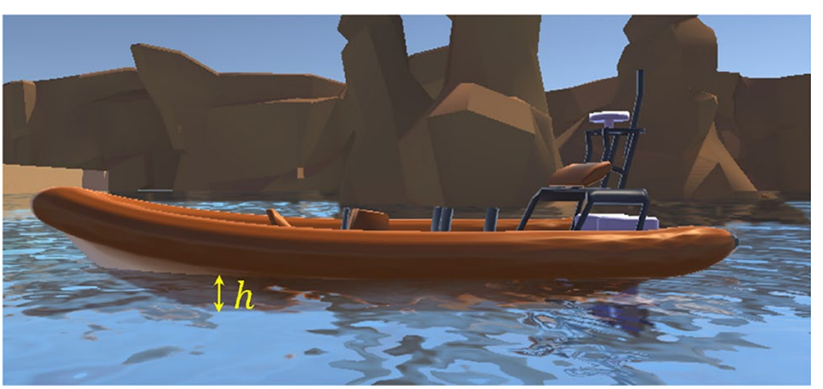

Fig. 12 Water level

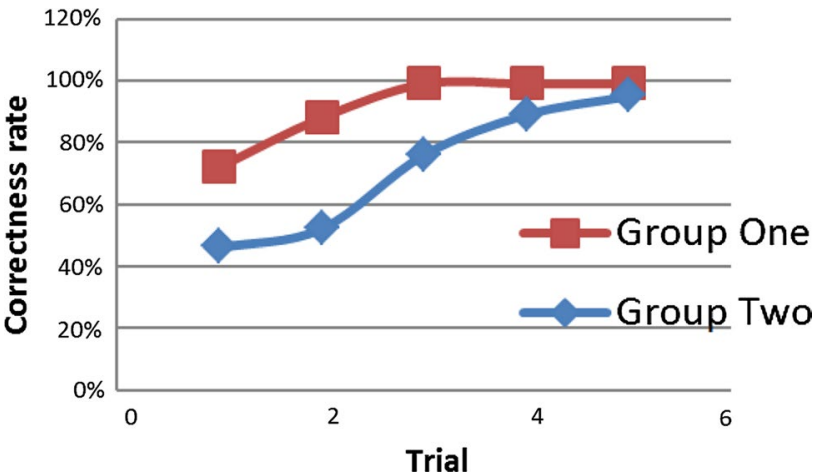

Fig. 13 Comparison of the correctness rate of the two groups

with $2.3 \%$ advantage of the average correctness. From the gradient of the line in Fig.12, it is clearly shown that Group One improves much faster than Group Two by trials. In conclusion, the participants who played our game achieved significantly better results than the ones who only read the manual. The participants in Group One were shown to have a deeper understanding of the whole process: they tried to analyse the reasons of these operation orders of the launching process rather than just remembering the manual context during the test. Figure 12 shows that after a few trials, both group perform identically. However, playing the game accelerates the training process since Group One performs equivalently good as Group Two but with only half of the time. The participants in Group One held positive attitudes towards the user experience of the game. They enjoyed the learning experience due to the interactivity and entertainment of the game.

We also did another experiment with the same participants of Group Two to gain more information about the performance of the players. All of the participants were asked to play the game for 8 trials within a limited time ( $5 \mathrm{~min}$ ). We tracked the average score each time. The results are plotted in Fig. 14. We found that the performance improved rapidly and players were able to avoid

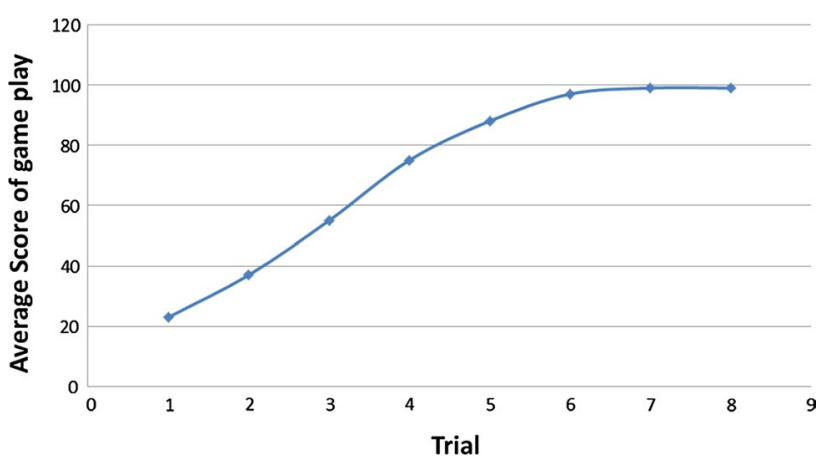

Fig. 14 Average scores over 8 games 
more potential hazards by practising. The players gave the credit of the performance improvement to the immersion of the game brought by the realistic physics of the game. The players were given the question of How do you feel about the game? Are these operations during the game intuitive? One of the player mentioned that the physical-corrected operation makes the game more realistic. Another one said the tractor drives in different speeds on different terrains, such as on slopes, that's really fun and I guess it gives the real-life feeling. Remembering the launching process and understanding the potential hazards is the first step into the real-life launching tasks. The game successfully turned a boring manual into a fun learning experience.

Therefore, the idea of using the game for the professional training in the future is very promising.

Reordering the launching process, though is far from enough knowledge to take real-life launching tasks, is a sensible and simple way to extract from a real-life task to a fun game. For the professional training purposes, the game still needs further development.

\section{Study 2: For safety awareness}

The game prototype not only serves the training purpose, but also helps to raise public awareness of the safety issues involved. Youth safety education is one of the core part of RNLI's prevention work (RNLI 2017c). Young people aged from 6 to 14 are the primary age period for youth safety education (RNLI 2017b) and for child health experiment (Bergeron et al. 2000). Therefore, we recruited 10 young participants ( 5 males and 5 females) for this case study, aged 6 to 14 .

This study focused mainly on the cognition of the potential hazards. As the same with Study 1, we separated them into two groups. Group One played our game, while Group Two did not. We designed a questionnaire to test the awareness of the potential hazards during a lifeboat launch. The participants from both groups answered the same questionnaire which consisted of 10 questions with questions like "What should you do when you need to cross the launch area, run across quickly or wait until the tractor has passed?". We gave every desired answer 10 points for each question. The average score of Group One was 84 , while the average score of Group Two was 72 which is $14.3 \%$ lower.

The participants who played our game performed better in the test of spotting and understanding the potential hazards which may happen during the lifeboat launch process. They also have a higher possibility to pay more attention to future safety issues when participating in seaside activities. Furthermore, the game also spreads the knowledge about a lifeboat launch.

The two studies show that the existing functions of the system have demonstrated the feasibility of our approach.
One of the participant stated "the game is entertaining as well as educational."

\section{VR game overview}

All the participants in study 1 were offered to experience the VR version of our game, and all of them gave positive feedbacks towards it. The VR version was considered to be more immersive and attractive than the non-VR one. They expressed that the VR game was more beneficial in learning about a lifeboat launch. They enjoyed the real-time feedback and real-like experiences from the game. It can be very helpful and useful for the training purposes. The only issue they raised is the dizziness caused by using the headset for a long time and the need to get used to the new manoeuvres control.

\section{Conclusions}

In this paper, we propose a game platform prototype for a lifeboat launch using educational and technical approaches. The virtual reality technology applied to a lifeboat launch provides real-time feedback and a real-like experience. It allows novices to acquire the experience of a lifeboat launch in a virtual environment without the risks in the real world and provides a much safer environment for users to get familiar with the operations.

The user evaluation results show that people learn better when they are actively engaged in acquiring and constructing knowledge in a learning-by-doing situation. The participants who played our game achieved significantly better than those who only read the manual. The game helps improve the performance rapidly. The participants also held positive attitude towards the user experience of the game which successfully turned a boring manual into a fun learning experience. Therefore, the idea of using the game for professional training purposes in future is very promising.

Furthermore, the game serves the educational purpose of awareness of the safety issues for general public while being entertaining. The case study of using the game for youth safety education successfully proves that the participants who played our game performed better in spotting and understanding the potential hazards during the lifeboat launch process.

Currently, we have gathered and analysed the data from the volunteers. Experiments which involve people with the skills of real-life launch are to be conducted in order to fully understand the effectiveness of the professional training and the extents to which the skills developed by playing the game transfer to real world.

The next goal of this work is to improve the visual design of the game and compare it with the current results to establish participant immersion quality. More tractor control can be added to improve the user experience. The driving mechanisms are rather simple. The graphics and the rendering 
quality of the game can be improved. Furthermore, the potential hazards in the situations are currently limited. With sufficient physical accuracy, the game could be developed as a test platform for different designs of a tractor model. The modularity property of the system architecture allows us to replace or add modules as a way to enhance particular features of the game. Another interesting add-on is to consider different weather conditions, such as a night, rain, wind, snow or heavy fog, which affect the players' visibility and add another level of challenge to the simulator.

Currently, users can interact using keyboard input or virtual reality devices but more advanced steering options can be experimented. According to the participants in the studies, the VR game is more immersive and interesting, and the knowledge of the lifeboat launch is gained while playing. However, VR devices still suffer several limitations. They are currently expensive. Most of the users cannot afford them in comparison to computers. VR devices are still undergoing further development to conquer the problem of dizziness especially when there is need to switch roles and viewpoints.

Acknowledgements The research leading to these results has received funding from the HEIF Fund (AANM02.X) Virtual Prototyping of New Lifeboat Launching System. The authors are grateful for the proofreading by Gabriel Notman and valuable inputs of anonymous reviewers.

Open Access This article is distributed under the terms of the Creative Commons Attribution 4.0 International License (http://creativecomm ons.org/licenses/by/4.0/), which permits unrestricted use, distribution, and reproduction in any medium, provided you give appropriate credit to the original author(s) and the source, provide a link to the Creative Commons license, and indicate if changes were made.

\section{References}

Abt CC (1987) Serious games. University Press of America, Lanham Aloni S, Khedkar S (2012) Comparative evaluation of tractor trolley axle by using finite element analysis approach. Int J Eng Sci Technol (IJEST) 4(4):1351-1360

Backlund P, Hendrix M (2013) Educational games-are they worth the effort? a literature survey of the effectiveness of serious games. In: 5th International conference on games and virtual worlds for serious applications (VS-GAMES), 2013, pp 1-8. https://doi. org/10.1109/VS-GAMES.2013.6624226

Baker IO (1919) A treatise on roads and pavements. Wiley, Hoboken Bergeron L, Valla JP, Breton JJ, Gaudet N, Berthiaume C, Lambert J, St-Georges M, Smolla N (2000) Correlates of mental disorders in the quebec general population of 6 to 14-year olds. J Abnorm Child Psychol 28(1):47-62

Brooks F (1999) What's real about virtual reality? IEEE Comput Graph Appl 19:16-27

Burdea GC, Coiffet P (2003) Virtual reality technology. IEEE. Wiley, Hoboken

CGM J (1994) Research on simulator-based training and instruction strategies. Train Simul 73-81
Epper RM, Derryberry A, Jackson S (2012) Game-based learning: developing an institutional strategy. EDUCAUSE Center for Applied Research, Research Bulletin, Louisville, CO

Fery YA, Ponserre S (2001) Enhancing the control of force in putting by video game training. Ergonomics 44(12):1025-1037

Gamberini L, Barresi G, Maier A, Scarpetta F (2008) A game a day keeps the doctor away: a short review of computer games in mental healthcare. J Cyber Ther Rehab 1(2):127-145

Gamberini L, Cottone P, Varotto D, Mantovani G (2010) Responding to a fire emergency in a virtual environment: different patterns of action for different situations. Ergonomics 46:842-858

Gee JP (2003) What video games have to teach us about learning and literacy. Comput Entertain (CIE) 1(1):20-20

Gillespie TD (1992) Fundamentals of vehicle dynamics. Technical report. SAE Technical Paper

Goldstone W (2009) Unity game development essentials. Packt Publishing Ltd, Birmingham

Gopher D, Well M, Bareket T (1994) Transfer of skill from a computer game trainer to flight. Human Factors J Human Factors Ergon Soc 36(3):387-405

Gredler ME (2004) Games and simulations and their relationships to learning. Handbook Res Edu Commun Technol 2:571-581

Greitzer FL, Kuchar OA, Huston K (2007) Cognitive science implications for enhancing training effectiveness in a serious gaming context. J Edu Resour Comput (JERIC) 7(3):2

Hibbeler R (2007) Engineering mechanics: statics \& dynamics, 11th edn. Prentice Hall, Upper Saddle River

Jonassen DH (1999) Designing constructivist learning environments. Instr Des Theor Models New Paradig Instr Theor 2:215-239

Lebram M, Engström H, Gustavsson H (2006) A driving simulator based on video game technology. In SIGRAD 2006. The Annual SIGRAD Conference; Special Theme: Computer Games. Linköping University Electronic Press

Macmillan RH (2002) The mechanics of tractor-implement performance. University of Melbourne, Melbourne.

Menard M (2011) Game development with Unity. Cengage Learning, Boston

Michael DR, Chen SL (2005) Serious games: games that educate, train, and inform. Muska \& Lipman/Premier-Trade, New York

National Research Council (US) (2006) Tires and passenger vehicle fuel economy: informing consumers, improving performance, vol 286. Transportation Research Board. Committee for the National Tire Efficiency Study, Transportation Research Board

Östlund J, Nilsson L, Törnros J, Forsman $\AA$ (2006) Effects of cognitive and visual load in real and simulated driving

Oztel I, Oz C (2014) Developing a virtual driving simulator for educational purposes. Balkan J Electr Comput Eng 2(2):51-54

RNLI (2017a) How we keep beaches safe. https://rnli.org/what-we-do/ lifeguards-and-beaches/how-we-keep-beaches-safe

RNLI (2017b) Teaching packs. https://rnli.org/youth-education/educ ation-resources/teaching-packs

RNLI (2017c) Youth education. https://rnli.org/youth-education

Rosser J, Lynch PJ, Haskamp LA, Yalif A, Gentile DA, Giammaria L (2004) Are video game players better at laparoscopic surgical tasks. In: Newport Beach, CA: Medicine meets virtual reality conference

Salen K, Zimmerman E (2004) Rules of play: Game design fundamentals. MIT press, Cambridge

Schneider W (1985) Training high-performance skills: fallacies and guidelines. Human Factors J Human Factors Ergon Soc 27(3):285-300

Shermann WR, Craig AB (2003) Understanding virtual reality. Morgan Kaufmann, Burlington

Susi T, Johannesson M, Backlund P (2007) Serious games: an overview

Unity (2017) Unity 2017: The world-leading creation engine for gaming. https://unity3d.com/unity 
Wolffelaar P, Bayarri S, Coma I (1999) Script-based definition of complex scenarios. In: Proceedings of the 4 th driving simulation conference, pp 7-8

Yann T (2016) 4 benefits of a game-based learning software for your company. https://elearningindustry.com/4-benefits-game-base d-learning-software-company
Youngblut C (1998) Educational uses of virtual reality technology. Technical report, DTIC Document 